\title{
Preacquisition REM sleep deprivation inhibits the formation of conditioned feeding suppression in rats
}

\author{
RICHARD L. SUTTON, WILLIAM TOMLINSON, and ROBERT A. HICKS \\ San Jose State University, San Jose, California 95192
}

\begin{abstract}
Forty male Wistar rats were divided into control and REM sleep-deprived groups to test the effects of REM deprivation on the acquisition of conditioned feeding suppression. Contrary to a theory that states that "prepared" learning is REM independent, we found that REM deprivation prevents the acquisition of conditioned feeding suppression.
\end{abstract}

To explain a set of equivocal findings that were generated by studies designed to measure the effects of pre- and postacquisition REM sleep deprivation (RD) on learning, Pearlman and his colleagues (Greenberg \& Pearlman, 1974; Pearlman, 1979; Pearlman \& Becker, 1974) postulated that slowly acquired, difficult tasks (i.e., unprepared learning) are REM dependent, whereas quickly acquired tasks (i.e., prepared learning) are REM independent. Thus, they have argued that acquisition of the former is seriously disrupted by RD, whereas acquisition of the latter is unaffected by RD.

Contrary to this hypothesis, it has been reported (Danguir \& Nicolaidis, 1976; Venkatakrishna-Bhatt, Bures, \& Buresova, 1978) that preacquisition RD inhibited the learning of conditioned taste aversion (CTA) in rats. That is, $R D$ interfered with the acquisition of a habit that is thought to be an example of prepared learning. While these studies seem to challenge Pearlman's REM-learning hypothesis, they may both be confounded in a manner that precludes this interpretation. To elaborate, Danguir and Nicolaidis (1976) claimed to have maintained their animals on a restricted drinking schedule (a condition necessary for CTA acquisition) while the animals were being REM deprived using the water-tank procedure. They argued that adulteration of the water in the $\mathrm{RD}$ apparatus with $.2 \%$ quinine prevented drinking. We tested this assumption in a pilot study and found that after a 2-day period of restricted drinking, our rats drank an average of $4.1 \mathrm{~g}$ of a $2 \%$ quinine-sulphate solution during a 5-min drinking test. Thus, Danguir and Nicolaidis' results may have reflected their failure to adequately control the drinking of their RD animals.

Venkatakrishna-Bhatt et al. (1978) attempted to correct this flaw in Danguir and Nicolaidis' (1976) procedure by using an RD method in which rats were placed on plastic cylinders surrounded by an electrified grid floor rather than water, as is the case in the com-

Address reprint requests to Robert A. Hicks, Department of Psychology, San Jose State University, San Jose, California 95192. This research was supported by NIH Grant RR08192-03. monly used water-tank RD procedure. While this change in methodology resolved the problem that may have confounded the Danguir and Nicolaidis study, it created another difficulty that may have confounded their study. To explain, it is well established that the watertank method of RD, if used correctly, is not stressful (e.g., see Fishbein \& Gutwein, 1977). However, since Venkatakrishna-Bhatt's group used a novel RD procedure that involved the use of electroshocks, it is clearly possible that their RD animals were differentially stressed by exposure to their novel RD apparatus. While it is difficult to argue that the results of this study can be explained as stress effects, the possibility exists that their results were confounded by these effects. Thus, both of these studies provide, at best, ambiguous evidence for the assertion that, contrary to Pearlman's hypothesis, preacquisition RD prevents learning of CTA.

The purpose of this research was to clarify the role of preacquisition RD in CTA and, in so doing, to provide evidence relevant to the validation of Pearlman's RD prepared and unprepared learning hypothesis. In this regard, as Venkatakrishna-Bhatt et al. (1978) have pointed out, previous research with bilateral functional decortication, cerebral anoxia, anesthesia, and hypothermia indicates that the most susceptible link in CTA acquisition is the initial processing of taste cues and the formation of a short-term gustatory trace. In view of the tentative findings of the aforementioned studies, we hypothesized that preacquisition RD would prevent the formation of a prepared response that does not involve drinking. Specifically, we measure the effects of $\mathrm{RD}$ on conditioned feeding suppression (CFS) in rats, when exposure to a novel-flavored food for a 1 -h period was followed by an intraperitoneal injection of $\mathrm{LiCl}$.

\section{METHOD}

\section{Animals}

The animals were 40 male Wistar rats that were 64 days old at the start of the experiment (weight range $=217-330 \mathrm{~g}$ ). These animals were divided into four groups $(\mathrm{N}=10 /$ group $)$ that were matched as closely as possible on the basis of body weight. In 
turn, these groups were randomly assigned to the four treatment conditions: 4 days of RD (SP) or one of three control conditions, a large-platform control condition (LP) or one of two dryconfinement control groups (DC1 or DC2).

Throughout the experiment, the animals were maintained on a 12-h/12-h light-dark cycle, with lights on at $0800 \mathrm{~h}$, in a room that was a nearly constant $22^{\circ} \mathrm{C}$.

\section{REM Deprivation}

RD was achieved using a modified version, for rats, of the water-tank procedure (see Fishbein \& Gutwein, 1977, for a critique of this procedure). Our RD apparatus and procedures have been described in detail elsewhere (e.g., see Hicks \& Moore, 1979). During the treatment period, all of the animals were confined in the RD apparatus (i.e., modified 18.9-liter buckets) for a 4-day period. Each animal in the SP group spent this time on a small platform (diameter $=6.5 \mathrm{~cm}$ ), which was surrounded by water, and thus was REM deprived. The LP group spent the treatment period on large platforms (diameter $=16.5 \mathrm{~cm}$ ) that were surrounded by water. These platforms were large enough to prevent the animals' falling into the water at REM onset, and thus this group acted as a non-REM-deprived control for exposure to the wet environment. Finally, each animal in the DC1 and DC2 groups spent the treatment period in a dry RD apparatus with the large platform in place. Therefore, a function of these groups was to serve as controls for exposure to the novelty of the RD apparatus.

\section{Procedure}

All animals were 33 days old upon arrival to our laboratory. During a 30-day adaptation period to the laboratory environment, animals were housed in individual wire-mesh cages with food and water available ad lib. Animals were gentled by handling for a 5-min period each day during this adaptation period.

After being weighed and assigned to treatment conditions on the 1st day of the experiment, animals were confined to buckets appropriate to their experimental condition. For the first 3 days of the experiment, food and water remained available ad lib. At the end of Day 3, food was removed from buckets and all animals were food deprived for a 24 -h period. After being weighed at the end of their 4th day in the buckets, animals were placed into individual, clear plastic mouse cages that were covered with metal feeding tops. Each mouse cage contained approximately $9 \mathrm{~g}$ of a novel-flavored food (oat and honey granola). Animals were allowed 1-h access to the novel food, during which they were closely monitored and prevented from falling asleep. At the end of this first feeding period, animals in the SP, LP, and DC1 groups were injected intraperitoneally with a dose of $6 \mathrm{ml} / \mathrm{kg}$ of $\mathrm{LiCl}(.15 \mathrm{M})$. The DC2 animals were injected intraperitoneally with an equivalent volume of isotonic saline. Immediately following injection, animals were returned to their home cages and given access to drinking water, which had not been available during the 1 -h feeding period. Two hours later, regular Lab Chow was returned to all animals.

For the next 3 days, all animals were maintained in their home cages with food and water available ad lib. At the end of Day 7 , food was removed from feeders and animals were food deprived for a 24-h period. On Day 8, animals were once again placed in the mouse cages for their second 1-h access to the oat and honey granola. Animals were prevented from falling asleep during the feeding period, and after this 1-h period, they were returned to their home cages, where food and water were provided.

\section{RESULTS AND DISCUSSION}

The respone measure in this experiment was the amount of novel food (granola) consumed during each of the two posttreatment feeding test sessions. Food consumption was measured by weighing the granola before and after each test session for each animal. The mean amounts of food consumed by each group during each test session are presented in Table 1 .

To analyze the data summarized in Table 1, first, separate one-way analyses of variance were computed for each feeding test, with these results: For the preinjection test, $F(3,36)=.26$ (n.s.), and for the postinjection test, $F(3,36)=5.67(\mathrm{p}<.01)$. To further elaborate the significant postinjection test results, several planned comparisons (see Keppel, 1982) were computed, with these results. The LP and DC1 group means were not significantly different (i.e., $F=.20$ ). This was also the case for the SP and DC2 (the saline control) group means $(F=.35)$. However, the differences between the SP group and the LP and DC1 groups and the DC2 group and the LP and $\mathrm{DC} 1$ groups were both significant $[\mathrm{F}(1,28)=13.46, \mathrm{p}<.001$, and $\mathrm{F}(1,28)=8.88, \mathrm{p}<.01$, respectively].

The implications of these results are clear. The LP and DC1 groups developed CFS, and the SP group did not. This conclusion is also supported by the analyses of the differences between the feeding test means for each group, which are listed in Table 1. Both the LP and DC1 groups showed a significant decrease in the amount of the novel food consumed (i.e., CFS), whereas the SP group showed a significant increase in the amount of the novel food consumed during the postinjection feeding test. Thus, the SP group that had been injected with $\mathrm{LiCl}$ behaved like the DC2 saline control group by showing no evidence of the development of CFS. Our results are consistent with those reported by Danguir and Nicolaidis (1976) and Venkatakrishna-Bhatt et al. (1978) and were derived using experimental procedures that we felt corrected apparent flaws in the design of each of these studies. Thus, it seems fair to conclude that RD prevents the formation of CFS.

If the acquisition of CFS is an example of "prepared learning" (we believe this to be the case), then these data constitute an important exception to the Pearlman REM-learning hypothesis, which states that prepared learning is REM independent. In a fairly recent paper, Pearlman (1979) discussed the findings of Danguir and

Table 1

Mean Food Consumption (in Grams) and the Standard Deviations for Each Treatment Group for Both Feeding Tests, and the ts Between the Test Means for Each Group

\begin{tabular}{|c|c|c|c|c|c|c|c|c|}
\hline \multirow{2}{*}{$\begin{array}{c}\text { Feeding } \\
\text { Test }\end{array}$} & \multicolumn{2}{|c|}{ SP } & \multicolumn{2}{|l|}{ LP } & \multicolumn{2}{|c|}{ DC1 } & \multicolumn{2}{|c|}{ DC2 } \\
\hline & Mean & SD & Mean & SD & Mean & SD & Mean & SD \\
\hline Preinjection & 1.2 & .4 & 1.1 & .4 & 1.3 & 1.0 & 1.4 & 1.3 \\
\hline Postinjection & 1.8 & 1.2 & .6 & .8 & .4 & .5 & 1.6 & 1.1 \\
\hline$t$ & $1.85^{*}$ & & $-1.85^{*}$ & & $-3.25 * *$ & & $.37 \dagger$ & \\
\hline
\end{tabular}

${ }^{*} p<.05 . \quad * * p<.01 . \quad$ tNonsignificant. 
Nicolaidis (1976) and Venkatakrishna-Bhatt et al. (1978) and called them "puzzling." We feel that when our results are considered together with these studies, the assertion that prepared learning is REM independent is no longer a tenable general hypothesis.

\section{REFERENCES}

Danauir, J., \& Nicolaidis, S. Impairments of learned aversion acquisition following paradoxical sleep deprivation in the rat. Physiology \& Behavior, 1976, 17, 489-492.

Fishbein, W., \& Gutwein, B. M. Paradoxical sleep and memory storage processes. Behavioral Biology, 1977, 19, $425-464$.

Greenbero, R., \& Pearlman, C. Cutting the REM nerve: An approach to the adaptive role of REM sleep. Perspectives in Biology and Medicine, 1974, 17, 513-521.
Hicks, R. A., \& Moore, J. D. REM sleep deprivation diminishes fear in rats. Physiology \& Behavior, 1979, 22, 689-692.

KE PPEL, G. Design and analysis (2nd ed.). Englewood Cliffs, N.J: Prentice-Hall, 1982.

Pearlman, C. A. REM sleep and information processing: Efidence from animal studies. Neuroscience and Biobehavioral Reviews, 1979, 3, 57-68.

Pearlman, C. A., \& Becker, M. REM sleep deprivation impairs bar-press acquisition in rats. Physiology \& Behavior, 1974, 13, 813-817.

Venkatakrishna-Bhatt, H., Bures, J., \& Buresova, O. Differential effect of paradoxical sleep deprivation on acquisition and retrieval of conditioned taste aversion in rats. Physiology \& Behavior, 1978, 20, 101-107.

(Received for publication November 17, 1982.) 